or by treatments for depression, is because such stimuli activate neuronal molecular signalling pathways. These pathways overlap with each other and with the signalling pathways that lead to dendritic structural changes.

Reid, I. C. \& Stewart, C. A. (200I) How

antidepressants work. New perspectives on the

pathophysiology of depressive disorder. British Journal of Psychiatry, 178, 299-303.

Vaidya, V. A. \& Duman, R. S. (200I) Depression emerging insights from neurobiology. British Medical Bulletin, 57, 61-79.

Yuste, R. \& Bonhoeffer,T. (200I) Morphological changes in dendritic spines associated with long-term synaptic plasticity. Annual Review of Neuroscience, 24, I07I-1089.

S. H. Zaman Mental Health Unit, Gloucester House, Southmead Hospital, Bristol BSI0 5NB, UK

R. Zaman Bedfordshire and Luton Community NHS Trust, Mental Health Service, Bedford, UK

Authors' reply: It was kind of Drs S. H. \& $\mathrm{R}$. Zaman to take an interest in our paper. In their thoughtful response they draw attention to the time course of LTP induction (seconds to minutes), and point out that this does not correlate with the time required for the effects (presumably, clinical response) of antidepressant treatments. The key issue is not the speed with which LTP induction itself occurs, which is unchanged by stress or antidepressant treatments (Stewart \& Reid, 1993). It is rather the time course of changes induced in the regulation of LTP by antidepressant treatments (the so-called 'metaplasticity' referred to in our paper) that is important. This develops gradually, requiring at least six spaced ECT treatments for maximum effect (Stewart et al, 1994) or 14 days of fluoxetine treatment (Stewart \& Reid, 2000). Interestingly, the effects of ECT on the degree to which LTP can be induced are detectable even 40 days after the end of a course (Stewart $\&$ Reid, 2000). These periods each correlate very nicely with antidepressant response, with the last described also mirroring the time course of relapse after successful ECT treatment in humans without antidepressant prophylaxis. Changes in excitatory post-synaptic potentials are seen, however, immediately after a single electroconvulsive application in experimental studies (Stewart et al, 1994), but they are smaller and more transient than those seen after a series of applications. This also accords with clinical observation: severely ill patients receiving ECT often show clear but transient responses after the first treatment in a course.

Of course, these are electrophysiological observations, and they may be mediated by ultrastructural neuronal changes. In this sense, we agree with the subtle point being made by Zaman \& Zaman. Our aims are to draw together rather than disaggregate structural and functional phenomena. That is why we used the term connectivity in the review to refer to both functional and ultrastructural (e.g. dendritic) changes underlying the plasticity of neuronal connections, which we wished to distinguish from more gross effects such as cell death or proliferation. The fact that "molecular signalling pathways" to "dendritic structural changes" and to LTP overlap is precisely why we classed them together as candidate contributors to the neurobiology of depressive disorder. They may be dissociable, as Zaman $\&$ Zaman point out, but this is not in itself evidence for or against the role of the regulation of LTP in affective disorder.

In any event the functional (electrophysiological plasticity) and structural changes (microanatomical plasticity) described in our review are each associated in reciprocal fashion with stress and antidepressant treatments, respectively - neither structural nor functional changes have been shown to have a causal role in depressive disorder. It does not allow that either phenomenon is a prerequisite for depressive states.

Stewart, C. \& Reid, I. (1993) Electroconvulsive stimulation and synaptic plasticity in the rat. Brain Research, 620, |39-|4|.

— $\mathbf{\&}$ - (2000) Repeated ECS and fluoxetine administration have equivalent effects on hippocampa synaptic plasticity. Psychopharmacology, 148, 217-223.

—, Jeffrey, K. \& Reid, I. C. (1994) LTP-like synaptic efficacy changes following electroconvulsive stimulation. Neuroreport, 5, 1041-1044.

I. Reid, C. Stewart Department of Psychiatry, University of Dundee, Ninewells Hospital, Dundee DDI 9SY, UK

\section{Leptin and antipsychotic drugs}

There is growing interest in the role of leptin in excessive body weight gain during antipsychotic drug treatment. Herrán et al's (2001) paper is an important contribution to the field. Among other interesting findings they demonstrated that the functioning of the leptin system is preserved during antipsychotic drug administration. However, it is not "the first study analysing the effects of chronic antipsychotic medication on serum leptin levels", since other authors have published relevant data for humans and rats (Baptista et al, 2000; Lacruz et al, 2000; Melkerson et al, 2000).

An important finding by Herrán et al was that olanzapine- and risperidonetreated patients displayed the highest and lowest leptin levels, respectively, "even after controlling for BMI". This may support the contention that olanzapine is promoting a deleterious metabolic profile. That finding also prompts speculation that other mechanisms besides body weight gain could be involved in leptin elevation during antipsychotic treatment.

We have proposed elsewhere that insulin may be one of these additional mechanisms. Insulin is a powerful stimulus for leptin synthesis and secretion. Female rats with sulpiride-induced obesity unexpectedly displayed normal serum leptin (and insulin) levels (Lacruz et al, 2000). In addition, serum leptin and insulin levels correlated positively in healthy people and antipsychotic-treated patients (Baptista et al, 2000, 2001). As olanzapine strongly stimulates appetite, it may promote insulin (and leptin) secretion, with relative independence from body weight gain. Surprisingly, Herrán et al reported that treatment with clozapine (another agent with strong appetite-stimulating properties) was associated with leptin levels similar to those found in haloperidol- and phenothiazine-treated patients (in spite of a higher body weight gain). If it were possible for Herrán et al to furnish the information, readers would benefit from knowing (a) the insulin levels in these patients; (b) a comparison of leptin levels between clozapine-, olanzapine- and risperidone-treated patients and their specific matched controls; and (c) the gender distribution in these treatment groups. If olanzapine- and risperidonetreated subjects display higher and lower leptin levels, respectively, than their controls, and if the gender distribution is similar in the three treatment groups, an additional important contribution will have been brought to the field of psychopharmacology.

Baptista, T., Lacruz, A., Silvera, R., et al (2000) Body weight gain after administration of antipsychotic drugs: correlation with leptin, insulin and reproductive hormones. Pharmacopsychiatry, 33, 81-88.

_, _, Angeles, F., et al (200I) Endocrine and metabolic profile in the obesity associated with typical antipsychotic drug-administration. Pharmacopsychiatry, in press. 
Herrán, A., García-Unzueta, M. T., Amado, J. A., et al (200I) Effects of long-term treatment with antipsychotics on serum leptin levels. British Journal of Psychiatry, 179, 59-62.

Lacruz, A., Baptista, T., de Mendoza, S., et al (2000) Antipsychotic drug-induced obesity in rats: correlation between leptin, insulin and body weight during sulpiride treatment. Molecular Psychiatry, 5, 70-76.

Melkerson, K. I., Hulting, A. L. \& Brismar, K. E. (2000) Elevated levels of insulin, leptin, and blood lipids in olanzapine-treated patients with schizophrenia or related psychoses. Journal of Clinical Psychiatry, 61, 742-749.

\section{Declaration of interest}

T.B. is currently a visiting scientist at McGill University, Montreal, and in this capacity is supported by Pfizer Lab, Canada.

T. Baptista, S. Beaulieu Douglas Hospital Research Center, 6875 Lasalle Blvd, Verdun, Québec, Canada $\mathrm{H} 4 \mathrm{HIR} 3$

\section{Gender differences in treatment response to antidepressants}

Parker (2001) expertly reviews the evidence for efficacy and effectiveness of different classes of antidepressants. In particular, he addresses the issue of whether selective serotonin reuptake inhibitors (SSRIs) are less efficacious in melancholia; reviews effectiveness studies; examines why there is a discordance between efficacy studies and clinical observation; and reviews the implications of differential effectiveness.

An additional important area that should be considered is that of gender differences in treatment response to antidepressants. Recent evidence has arisen to suggest that women may have a better response to SSRIs and men a better response to tricyclic antidepressants (Kornstein et al, 2000). As depression is approximately twice as common in women than in men, gender differences of this nature are important and should be considered when reviewing this area. The reasons for this difference may be related to the effects of female sex hormones on serotonergic neurotransmission and in particular on the function of the $5-\mathrm{HT}_{1 \mathrm{~A}}$ receptor (Young et al, 1993). The evidence for gender differences in treatment response to antidepressant is not definitive (Kornstein et al, 2001; Quitkin et al, 2001) and further studies are needed to fully establish the validity of this notion. However, there are potentially important implications, and in the future clinical management strategies may take account of gender differences in treatment response.

Kornstein, S. G., Schatzberg, A. F., Thase, M. E., et al (2000) Gender differences in treatment response to sertraline versus imipramine in chronic depression. American Journal of Psychiatry, 157, 1445-1452.

_ , _, , et al (200I) Dr Korstein and Colleagues reply (letter). American Journal of Psychiatry, 158, 1532-1533.

Parker, G. (200I) 'New' and 'old' antidepressants: all equal in the eyes of the lore? British Journal of Psychiatry, 179, 95-96.

Quitkin, F. M., Stewart, J. W. \& McGrath, P. J. (200I) Gender differences in treatment response (letter). American Journal of Psychiatry, 158, 1531-1532.

Young, A. H., Dow, R. C., Goodwin, G. M., et al (1993) The effects of adrenalectomy and ovariectomy on the behavioural and hypothermic responses of rats to 8 hydroxy-2(di-n-propylamino)tetralin (8-OH-DPAT). Neuropharmacology, 32, 653-657.

\section{Declaration of interest}

I have received speakers' fees from pharmaceutical companies that manufacture SSRIs.

A. H. Young Department of Psychiatry, The Roya Victoria Infirmary, Queen Victoria Road, Newcastle upon Tyne, NEI 4LP, UK

\section{World psychiatric literature}

Patel \& Sumathipala (2001) have provided an interesting survey of the country of origin of papers published in six psychiatric journals over a 3 -year period. Only $6 \%$ of the literature was published from nonEuro-American countries. Unfortunately, the authors equate this low representation with the state of international research in psychiatry and these inferences are amplified by two accompanying commentaries (Leff/Cheng, 2001).

It has been repeatedly shown that use of journals' impact factors to infer the actual citations of individual papers or investigators is misleading (Fava \& Ottolini, 2000). Further, both the authors of and the commentators on the study have failed to cite the research evidence available in terms of international trends (Fava \& Montanari, 1998). Such evidence is based on the National Science Indicators on Diskette developed by the Institute for Scientific Information. All journals listed in Current Contents under the heading of Psychiatry were included in the database. The number of papers published per year, their citations, and the number of citations received per paper published were reported. Even though North America and the European
Union rule the psychiatric arena, the picture is rather different from the one portrayed by Patel \& Sumathipala. In the most recent survey of world psychiatric literature (Fava et al, 2001), limited to the journals included under the heading of Clinical Psychology and Psychiatry in Current Contents (Clinical Medicine), an impressive growth in impact from Latin America was documented. Fortunately, the progress of international psychiatric research is not confined to the six journals selected for the analysis by Patel \& Sumathipala. None the less, many of their comments are valid and useful.

Fava, G. A. \& Montanari, A. (1998) National trends in behavioral sciences (198I-1996). Psychotherapy and Psychosomatics, 67, 28I-30I.

\& Ottolini, F. (2000) Impact factors versus actu citations. Psychotherapy and Psychosomatics, 69, 285-286.

_, _ \& Sonino, N. (2000I) Which are the leading countries in clinical medicine research? A citation analysis (1981-1998). Psychotherapy and Psychosomatics, 70, 283-287.

Leff, J./Cheng, A. T. A. (200I) Invited commentaries on: International representation in psychiatric literature. Survey of six leading journals. British Journal of Psychiatry I78, 410-4II.

Patel,V. \& Sumathipala, A. (2000I) International representation in psychiatric literature. Survey of six leading journals. British Journal of Psychiatry, I78, 406-409.

G. A. Fava, F. Ottolini, E. Tossani Department of Psychology, University of Bologna, Viale Berti Pichat 5, 40127 Bologna, Italy

\section{Error in Andrews et al (200I)}

Dr Meadows and colleagues have drawn our attention to a wrong and misleading sentence in the Method section of Andrews et al (2001a). We conclude the sub-section on assessment (p. 146) by saying "Perceived health need was based on the UK Survey of Psychiatric Morbidity questions". This sentence should read: "Perceived health need was based on work by Meadows et al (2000a). Similar concepts were used by the UK Survey of Psychiatric Morbidity questions".

We apologise for this omission and draw readers' attention to Meadows et al (2000b) for a more complete discussion of the development of the Perceived Need for Care Questionnaire. This is a reference we have used in subsequent papers (e.g. Andrews et al, 2001b).

Andrews, G., Henderson, S. \& Hall, W. (200la) Prevalence, comorbidity, disability and service utilisation 